\section{Miliary Tuberculosis Following Negative Latent Tuberculosis Infection Screening Prior to Tumor Necrosis Factor- $\alpha$ Antagonists: Implications for Management?}

\section{To the Editor:}

Tumor necrosis factor- $\alpha$ (TNF- $\alpha)$ antagonists are effective treatments for inflammatory disorders but are associated with an increased risk of infections including reactivation of latent tuberculosis infection (LTBI). We describe a patient who developed miliary TB as a complication of TNF- $\alpha$ antagonist therapy despite negative pretherapy screening for LTBI.

A 42-year-old woman with severe psoriatic arthritis presented with a 3-week history of fevers, night sweats, nonproductive cough, and 4-kg weight loss. She had been receiving treatment with adalimumab for 3 years after failing other disease-modifying antirheumatic drugs. A computerized tomography scan of her chest showed widespread nodules, bilateral lower zone patchy consolidation, and moderate splenomegaly, with small areas of nonenhancement consistent with miliary TB (Figure 1). She underwent bronchoscopy, which was smear- and culture-positive for Mycobacterium tuberculosis. She started isoniazid, rifampicin, pyrazinamide, and ethambutol, with resolution of symptoms over the following 2 weeks.

This patient was born in Ireland and migrated to Australia when 3 months old. At age 12 years, her step-brother was diagnosed with pulmonary TB, her only known TB exposure. As part of family contact screening, she underwent tuberculin skin testing (TST), which was nonreactive, and subsequently received a Bacillus Calmette-Guerin vaccine. Prior to commencement of adalimumab, she was reinvestigated for latent TB infection with a chest radiograph, which was normal, and interferon- $\gamma$ release assay (IGRA; QuantiFERON ${ }^{\circledR}$-TB Gold In-Tube, Cellestis, Chadstone, Australia), which was negative. She had limited local travel, no international travel, and no other history to suggest new exposure to TB following the 2 screening tests. Although possible, this history makes new local acquisition of TB after screening unlikely. Following her symptomatic TB disease, a repeat IGRA was positive.
With increasing use of TNF- $\alpha$ antagonists, it has become apparent that this treatment is associated with an increased risk of serious infections. These are particularly due to intracellular pathogens, most notably a 5 -fold increase in the risk of reactivation of $M$. tuberculosis infection ${ }^{1,2}$. TNF- $\alpha$ antagonists carry differential risks, with infliximab and adalimumab having higher rates of TB reactivation than etanercept ${ }^{3}$. As highlighted by our case, adalimumab is associated with the longest median time to reactivation, and TB is more likely to be disseminated with this than with the other TNF- $\alpha$ antagonists ${ }^{3}$.

Screening for and treatment of LTBI prior to initiation of TNF- $\alpha$ antagonists has been shown to be effective in markedly reducing the rates of TB reactivation ${ }^{4}$. The Australian Rheumatology Association has published guidelines on screening for LTBI prior to use of TNF- $\alpha$ antagonists, which recommend case history risk factor assessment, chest radiograph, and an IGRA or 2-step TST. In these guidelines, patients with "high" risk factors should be referred for consideration of LTBI treatment, which generally consists of 9 months of isoniazid therapy ${ }^{5}$. Other guidelines have instead suggested that only patients with prior personal history of TB, changes on chest radiograph, or positive IGRA should be referred for further assessment and treatment ${ }^{6}$.

As illustrated by our case, prior negative testing for LTBI does not eliminate the risk of TB following commencement of TNF- $\alpha$ antagonist therapy. The patient had a history of a nonhousehold TB contact but TB screening tests had been negative on 2 occasions; first, immediately following TB contact in childhood, and second, prior to TNF- $\alpha$ antagonist therapy. Accordingly, she was not given treatment for LTBI.

The case illustrates some of the difficulties in TB risk factor assessment and the pitfalls of relying on screening tests that carry a risk of false-negative results, especially in the setting of immunosuppression ${ }^{7}$. Alternative approaches, such as serial LTBI testing after initiation of TNF- $\alpha$ antagonists, have been proposed, as rates of conversion from negative to positive as high as $30 \%$ have been reported ${ }^{8}$. However, the clinical implications of such strategies are unclear.

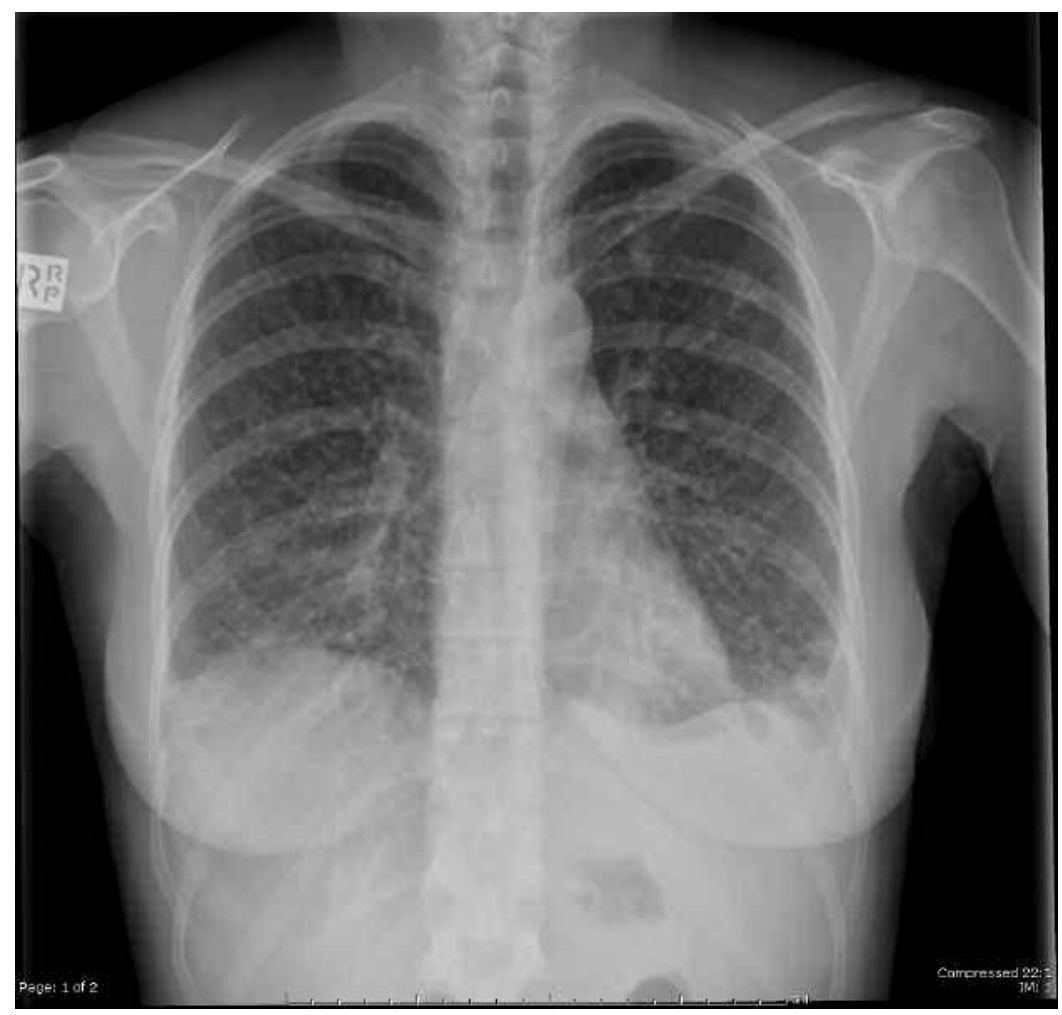

Figure 1. Chest radiograph of the patient shows disseminated nodules throughout both lung fields. 
This case highlights the need for careful specialist assessment of TB risks for all patients receiving TNF- $\alpha$ antagonist therapy. In addition to encouraging routine pre-TNF- $\alpha$ antagonist therapy screening, we would remind clinicians that high-risk patients, as defined by a personal history of TB (incompletely treated or not documented), unexplained abnormalities on chest radiograph in the appropriate epidemiological setting, or known contact with infectious pulmonary TB, should be considered for LTBI treatment regardless of the results of screening tests unless there is an unacceptably high risk of hepatotoxicity with isoniazid. A summary of practice points derived from this experience is given in Table 1. For patients born in high TB-incidence countries but without a defined history of TB exposure, we suggest that regular clinical monitoring for TB while on TNF- $\alpha$ antagonist therapy is appropriate, while alternative strategies such as serial IGRA or TST warrant further study. These cases illustrate that current screening strategies will not prevent all cases of TB reactivation and that clinicians need to maintain heightened vigilance for TB in patients receiving TNF- $\alpha$ antagonists, regardless of results of LTBI screening.

Table 1. Summary of practice points from the case.

Tumor necrosis factor- $\alpha$ (TNF- $\alpha$ ) inhibition is associated with an increased risk of tuberculosis (TB) reactivation

Pretreatment screening for latent tuberculosis infection (LTBI) substantially reduces but does not eliminate the risk of TB disease

Patients with a high risk of TB exposure may be considered for LTBI treatment even if screening tests are negative

Expert assessment of TB risk should be sought before commencement of TNF- $\alpha$ inhibition

BENJAMIN W. TEH, MBBS; ALAN C. STREET, MBBS, FRACP; EMMA S. McBRYDE, MBBS, FRACP, PhD; JUSTIN T. DENHOLM, MBBS, FRACP,

Victorian Infectious Diseases Service, Royal Melbourne Hospital, Grattan Street, Parkville 3050, Victoria, Australia. Address correspondence to Dr. B.W. Teh; E-mail: ben.w.teh@gmail.com.

The authors thank Associate Professor Damon Eisen for his contribution and review of this report.

\section{REFERENCES}

1. Galloway JB, Hyrich KL, Mercer LK, Dixon WG, Fu B, Ustianowski AP, et al. Anti-TNF therapy is associated with an increased risk of serious infections in patients with rheumatoid arthritis especially in the first 6 months of treatment: Updated results from the British Society for Rheumatology Biologics Register with special emphasis on risks in the elderly. Rheumatology 2011;50:124-31.

2. Ledingham J, Wilkinson C, Deighton C. British Thoracic Society (BTS) recommendations for assessing risk and managing tuberculosis in patients due to start anti-TNF-alpha treatments. Rheumatology 2005;44:1205-6.

3. Dixon WG, Hyrich KL, Watson KD, Lunt M, Galloway J, Ustianowski A, et al. Drug-specific risk of tuberculosis in patients with rheumatoid arthritis treated with anti-TNF therapy: Results from the British Society for Rheumatology Biologics Register (BSRBR). Ann Rheum Dis 2010;69:522-8.

4. Carmona L, Gomez-Reino JJ, Rodriguez-Valverde V, Montero D, Pascual-Gomez E, Mola EM, et al. Effectiveness of recommendations to prevent reactivation of latent tuberculosis infection in patients treated with tumor necrosis factor antagonists. Arthritis Rheum 2005;52:1766-72.

5. Australian Rheumatology Association. Screening for latent tuberculosis infection (LTBI) prior to the use of biological agents in Australia. Therapeutics Committee, Australian Rheumatology Association. April 2010. [Internet. Accessed March 7, 2012.] Available from: http://tbevidence.org/documents/ guidelines/Australian\%20TNF\%20screening\%202010.pdf

6. Gupta A, Street AC, Macrae FA. Tumour necrosis factor alpha inhibitors: Screening for tuberculosis infection in inflammatory bowel disease. Med J Aust 2008;188:168-70.

7. Pai M, Zwerling A, Menzies D. Systematic review: T-cell-based assays for the diagnosis of latent tuberculosis infection: an update. Ann Intern Med 2008;149:177-84.

8. Park JH, Seo GY, Lee JS, Kim TH, Yoo DH. Positive conversion of tuberculin skin test and performance of interferon release assay to detect hidden tuberculosis infection during anti-tumor necrosis factor agent trial. J Rheumatol 2009;36:2158-63.

J Rheumatol 2012;39:6; doi:10.3899/jrheum.111577 